\title{
Nature's Conveyor Belt: The Matrix Mediated Biomineralization of Magnetite in Chitons (Mollusca)
}

\author{
J. A. Shaw, ${ }^{*}$ D. J. Macey, ${ }^{* *}$ L. R. Brooker, ${ }^{* * *}$ M. Saunders, ${ }^{*}$ P. L. Clode ${ }^{*}$ \\ ${ }^{*}$ Centre for Microscopy, Characterisation and Analysis, University of Western Australia, Crawley, \\ Perth, WA 6009, Australia \\ ** School of Biological Sciences \& Biotechnology, Murdoch University, Murdoch, WA 6150, \\ Australia \\ **** Faculty of Science, Health and Education, University of the Sunshine Coast, Maroochydore DC, \\ QLD 4558, Australia
}

Chitons are marine molluscs that use a variety of iron and calcium based minerals to harden their teeth, which they use to scrape algae growing upon, and within, rocks. The teeth are mounted on a long ribbon-like organ termed the radula, with immature, unmineralized teeth at the posterior end and the hardened iron-mineralized teeth at the anterior end (Fig. 1). At any one time, up to 80 individual tooth rows can be observed, with each row becoming progressively mineralized as it moves forward in a conveyor belt-like manner. The ability to study the entire mineralization process in a single animal makes these creatures ideal for the study of matrix mediated biomineralization. The chiton's ability to mineralize iron has inspired researchers who believe that new biomimetic materials and technologies can be developed based on the principles of biomineral formation.

Two main aspects of the tooth mineralization process are under investigation. The first examines the cells that mediate the delivery of mineralizing elements to, and the solution chemistry within, the organic matrix scaffold of the tooth cusps. The second examines the matrix itself and the organization and types of minerals that precipitate on its surface. Electron microscopy (EM) is a fundamental tool for structural investigations of both the cellular and mineralized aspects of these biomineralizing tissues. A wide range of analytical EM techniques, including those facilitated by transmission electron microscopy (TEM) and scanning electron microscopy (SEM), have been employed to investigate the mechanisms of biomineralization in chiton teeth.

Energy-filtered TEM mapping reveals that iron, stored within the transport protein ferritin, is passed from the cells surrounding the teeth and into the tooth matrix via the microvilli (Fig. 2) [1]. Iron mineralization is initiated at tooth row 13 by the simultaneous influx of iron from the tissues and from the junction zone, a region identified as a storage site for mineralizing elements (Fig. 3) [2-3]. X-ray microanalysis, conducted using SEM, reveals that element transfer from the junction zone into the tooth cusp occurs via a plume situated within the tooth core (Fig. 3). We suggest that the coordinated delivery of elements from the cells and the junction zone into the teeth is necessary for the formation of these mineralogically complex structures.

References

[1] J. A. Shaw et al., Microsc. Microanal. (in press) (2009).

[2] D. J. Macey and L. R. Brooker, J. Morph. 230 (1996) 33.

[3] J. A. Shaw et al., J. Morph. (in press) (2009).

[4] Work supported by Australian Research Council Discovery Grant \# DP0559858 D. J. Macey 


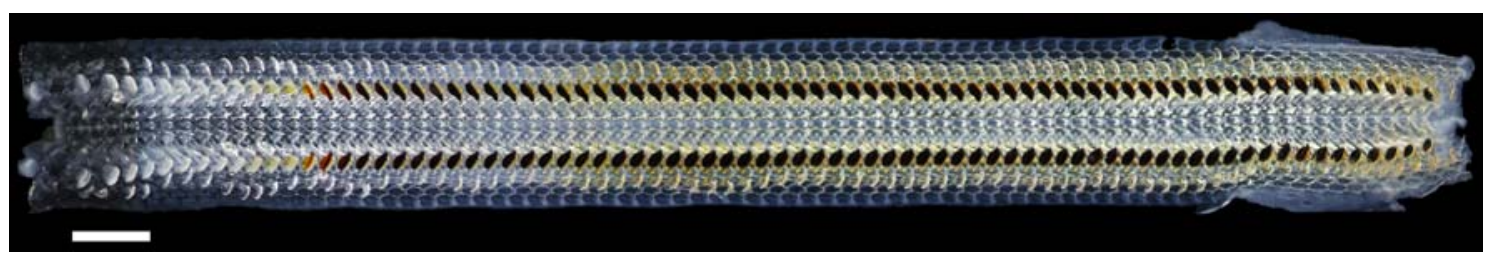

Fig. 1. Light micrograph of the radula feeding organ excised from the chiton Acanthopleura hirtosa. The teeth at the posterior end of the radula (left) start as an unmineralized organic scaffold (clear teeth), and as they move towards the anterior end become progressively mineralized with iron (black teeth). Scale bar $=1 \mathrm{~mm}$.

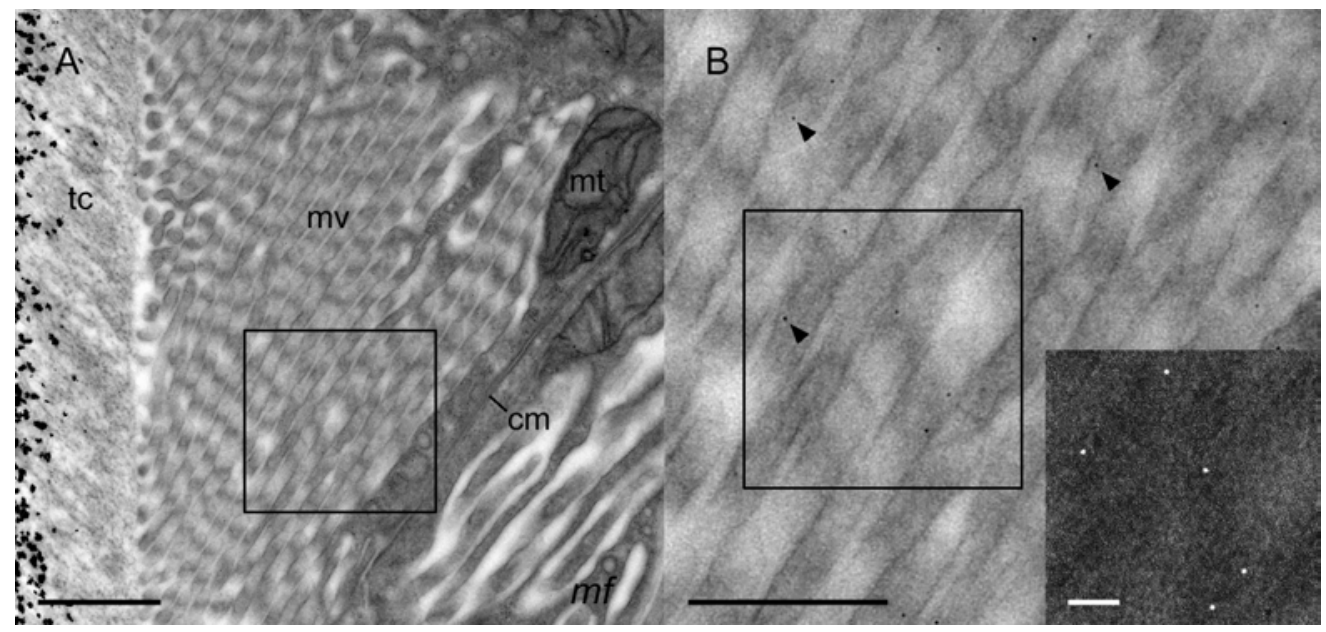

Fig. 2. TEM image of the epithelial cells associated with iron mineralization. A. The microvilli (mv), at the apical end of each cell, attach to the tooth cusp (tc) and numerous electron dense particles, characteristic of ferritin, can be observed near the cell membranes (cm) and mitochondria (mt). B. At higher magnification (square region in A), ferritin can be observed within the microvilli, as confirmed by the EFTEM iron map (inset). Scale bars $=$ (A) $1 \mu \mathrm{m}$, (B) $500 \mathrm{~nm}$, inset $100 \mathrm{~nm}$ From [1] with permission.
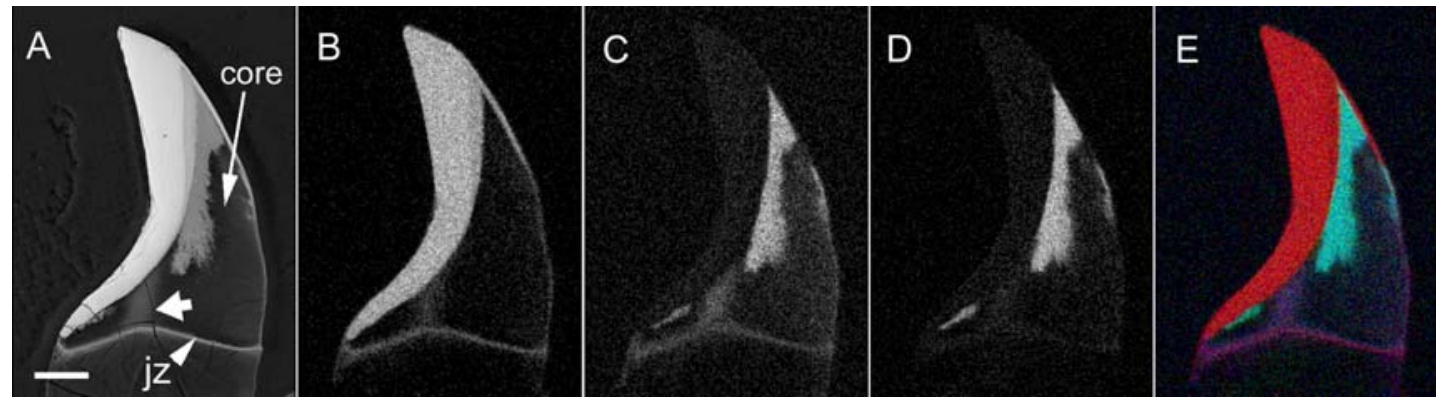

Fig. 3. SEM images of a single chiton tooth in longitudinal section during the process of apatite mineralization of the core. A. The back-scatter image shows a plume of elements (arrow) between the junction zone (jz) and posterior iron mineralized region. B-D. Element maps, obtained using energy-dispersive $\mathrm{x}$-ray microanalysis for B. iron, C. phosphorous and D. calcium, and the E. red (iron), green (phosphorous) and blue (calcium) composite color image, show that the plume is comprised of a combination of these three elements. Scale bar $=50 \mu \mathrm{m}$. 